\title{
Médiévales
}

Langues, Textes, Histoire

61 | automne 2011

La chair des émotions

\section{Les avatars de l'inquisiteur ou la fonction de juge ecclésiastique à la fin du Moyen Âge}

Laurence Silvestre

\section{(2) OpenEdition}

1 Journals

Édition électronique

URL : https://journals.openedition.org/medievales/6562

DOI : $10.4000 /$ medievales. 6562

ISSN : $1777-5892$

Éditeur

Presses universitaires de Vincennes

\section{Édition imprimée}

Date de publication : 20 décembre 2011

Pagination : 187-203

ISBN : 978-2-84292-337-2

ISSN : 0751-2708

\section{Référence électronique}

Laurence Silvestre, « Les avatars de l'inquisiteur ou la fonction de juge ecclésiastique à la fin du Moyen Âge », Médiévales [En ligne], 61 | automne 2011, mis en ligne le 21 janvier 2012, consulté le 23 avril 2022. URL : http://journals.openedition.org/medievales/6562 ; DOI : https://doi.org/10.4000/ medievales.6562 
Médiévales 61, automne 2011,p. 187-204

Laurence SILVESTRE

\section{LES AVATARS DE L'INQUISITEUR OU LA FONCTION DE JUGE ECCLÉSIASTIQUE À LA FIN DU MOYEN ÂGE}

En matière de foi, les juges ne sont pas toujours ce que l'on croit et l'habit ne fait pas forcément l'inquisiteur. Un juge peut même en cacher un autre. Quand on parle d'inquisitio, ou inquisition, c'est d'abord pour évoquer une procédure d'enquête et d'enregistrement de témoignages ${ }^{1}$. Dès la fin du $\mathrm{XII}^{\mathrm{e}}$ siècle, elle s'est imposée dans la justice ecclésiastique; elle fut en premier lieu le fait des évêques, juges ordinaires, puis, à partir de 1231, de délégués extraordinaires de la papauté qui prirent alors le titre de pravitatis hereticae inquisitores. Les inquisiteurs étaient nés, et avec eux l'inquisition dite pontificale. C'est cette inquisition qui a alimenté tant de fantasmes et dont certains ont tiré une légende noire. Cependant de nombreux travaux concernant les XIV et $\mathrm{XV}^{\mathrm{e}}$ siècles ont montré que l'inquisition médiévale est plus complexe que les stéréotypes habituels de moines fanatiques et d'implacable machine bureaucratique. Le médiéviste doit se garder de tels préjugés, et l'historiographie de ces dernières années est riche de recherches qui vont à rebours des clichés sur l'Inquisition, en soulignant notamment les liens avec le pouvoir ou le caractère pénitentiel des condamnations ${ }^{2}$. Dans les sources médiévales, le terme d'«inquisiteur» n'indique

1. Voir C. Gauvard éd., L’Enquête au Moyen Âge, Rome, 2008.

2. Sur les publications récentes, voir les collectifs: G. Audisıo (éd.), Inquisition et pouvoir,

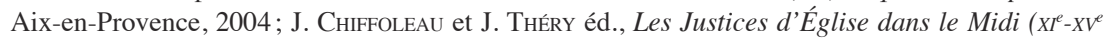
siècle), Toulouse, 2007 (Cahiers de Fanjeaux, 42); Praedicatores, Inquisitores, t. 1, The Dominicans and the Medieval Inquisition, Acts of the 1st International Seminar on the Dominicans and the Inquisition, 23-25 February 2002, Rome, 2004; B. LemesLe éd., La Preuve en justice de l'Antiquité à nos jours, Rennes, 2003, en particulier A. Provost, «Déposer, c'est faire croire? À propos du discours des témoins dans le procès de Guichard, évêque de Troyes (1308-1314)», p. 95-118 et J. THÉRY, «Fama: l'opinion publique comme preuve judiciaire. Aperçu sur la révolution médiévale 
d'ailleurs pas systématiquement le représentant appointé du pouvoir pontifical; il peut s'agir simplement de celui qui enquête. Et c'est toute la gageure du travail de l'historien de faire que soudain les individus incarnant l'officium inquisitionis sortent de l'obscurité, pour aider peut-être à redéfinir la charge qu'ils remplissent et saisir enfin la réalité de l'inquisitio. Peut-on parler de métier ou même de poste de juge ecclésiastique? Qui sont ces juges? Hommes de pouvoir? Hommes d'appareil? Hommes de main, voire hommes de paille?

Deux ouvrages récents présentent la figure du juge ecclésiastique et la répression de l'hérésie médiévale sous deux angles fort différents. Dans un cas, avec Le Prédicateur et l'Inquisiteur. Les tribulations de Baptiste de Mantoue à Genève en $1430^{3}$ de Franco Morenzoni, il s'agit de publier le dossier d'une procédure assez peu connue qui concerne la Suisse romande; dans l'autre, avec Pierre Cauchon. Comment on devient le juge de Jeanne d'Arc ${ }^{4}$ de Jean Favier, c'est une retentissante affaire nationale, maintes fois traitée, qui est envisagée comme point d'orgue du parcours d'un évêque passé à la postérité pour avoir condamné Jeanne d' $\mathrm{Arc}^{5}$. Ils ne sont l'un et l'autre, à première vue, pas fondamentaux pour l'histoire de «l'Inquisition», telle qu'on a tendance à se la représenter. Ils peuvent, néanmoins, stimuler la réflexion des historiens et nourrir une recherche sur l'inquisitio médiévale, qui est toujours d'actualité.

Depuis quelques décennies, en effet, les publications des historiens de la répression, des déviances et de la sorcellerie, témoignent des nombreuses

de l'inquisitoire (XII-XIV e siècle)»,p. 119-147. Voir également J. THÉRY, «"Inquisitio contre Rixende, fanatique du XIII siècle": la copie d'un document perdu des archives de l'archevêché de Narbonne par le Minime François Laporte (BM Toulouse, ms 625, f. 73-83, vers 1710)», M. FouRniÉ et D. LE BLÉvec éd., L'Archevêché de Narbonne au Moyen Âge, Toulouse, 2008, p. 63-90; ID., «Philippe le Bel, la persécution des "perfides Templiers" et la pontificalisation de la royauté capétienne», dans L'età dei processi. Inchieste e condanne trà politica e ideologia nel '300, Rome, 2009, p. 63-80; ID., «Atrocitas/enormitas. Per una storia della categoria di "crimine enorme" nel Basso medioevo (sec. XII-XV)», Quaderni storici, 131 (2009), p. 329-375 (texte remanié et augmenté dans l'article en ligne: "Atrocitas/enormitas. Pour une histoire de la catégorie d"“énormité" ou "crime énorme" du Moyen Âge à l'époque moderne», Clio@Themis, Revue électronique d'histoire du droit, 4 (2011), en particulier les pages consacrées à «l'arbitraire du juge», p. 25-34); ID., «Procès des Templiers», dans N. Bériou, P. Josserand éd., Prier et combattre. Dictionnaire européen des ordres militaires, Paris, 2009, p. 743-750; ID. éd., Le Livre des sentences de l'inquisiteur Bernard Gui (1308-1323), Paris, 2010.

3. F. Morenzoni (avec la collaboration d'I. JEger), Le Prédicateur et l'Inquisiteur. Les tribulations de Baptiste de Mantoue à Genève en 1430, Lyon, 2006.

4. J. FAVIER, Pierre Cauchon. Comment on devient le juge de Jeanne d'Arc, Paris, 2010.

5. Pour les pièces du procès de 1431, voir J. Quicherat, Procès de condamnation et de réhabilitation de Jeanne d'Arc, 5 vol., Paris, 1841-1848; pour celles du procès de 1455-1456, voir P. Duparc, Procès en nullité de la condamnation de Jeanne d'Arc, 5 vol., Paris, 1977-1988. La liste des publications concernant Jeanne et ses procès est longue, mais on pourra toujours se référer à A. et G. Duby, Les Procès de Jeanne d'Arc, Paris, 1973; C. Beaune, Jeanne d'Arc, Paris, 2004. 
variantes de la justice ecclésiastique au Moyen Âge. Toute une génération de chercheurs a adopté, en outre, une approche résolument micro-historique de la question, qu'on retrouve chez F. Morenzoni. Il s'agit dès lors d'étudier en détail des affaires précises, à la faveur de procès-verbaux ou de chroniques bien renseignées, permettant de saisir des pratiques locales et des hommes, ainsi que des normes et tout un imaginaire relatif aux crimes relevant de l'inquisition. Il en résulte des analyses minutieuses des documents en tant que sources d'informations mais aussi, et surtout, en tant qu'objets d'histoire et, dans de nombreux ouvrages, c'est une véritable déconstruction des procès qui est alors mise en œuvre ${ }^{6}$. L'époque des grandes synthèses n'est cependant pas révolue.

Dans le sillage des travaux de l'école de Lausanne ${ }^{7}$, le livre de F. Morenzoni se propose en effet d'étudier un épisode de l'histoire de l'inquisition suisse, en éditant et commentant un registre conservé à l'Archivio di Stato de Turin ${ }^{8}$. J. Favier opte, en revanche, pour une approche macro-historique en publiant une vaste fresque sous un titre apparemment monographique. Mais ce que ces deux ouvrages ont en commun n'est pas négligeable: les procédures d'inquisition qui constituent le point de mire de l'analyse minutieuse de F. Morenzoni d'une part, et le point de fuite du tableau de J. Favier d'autre part, sont contemporains, presque concomitants, puisque l'affaire Baptiste de Mantoue éclate en 1430 et que le procès de Jeanne d'Arc se déroule en 1431. À défaut de partager un contexte géographique, les deux affaires ont lieu à un moment critique de l'histoire de l'Occident médiéval, véritable tournant de l'histoire de l'Église; elles en portent l'empreinte. Dans les deux livres par ailleurs, on peut aussi voir une entreprise de condamnation du juge sur laquelle il faudra revenir. Ces publications diffèrent néanmoins dans leur méthode, leur style et leur sujet, et ne s'adressent pas aux mêmes lecteurs.

6. Voir F. Mercier, La Vauderie d'Arras. Une chasse aux sorcières à l'automne du Moyen Âge, Rennes, 2006 (tiré de sa thèse soutenue à l'Université Lumière Lyon 2: L'Enfer du décor. La Vauderie d'Arras (1459-1491) ou l'émergence contrariée d'une nouvelle souveraineté autour des ducs Valois de Bourgogne); F. Provost, Domus Diaboli. Un évêque en procès au temps de Philippe le Bel, Paris, 2010.

7. Voir notamment Médiévales, 44 (2003); M. Ostorero, K. Utz Tremp éd., G. Modestin collab., Inquisition et sorcellerie en Suisse romande. Le registre Ac 29 des Archives cantonales vaudoises (1438-1528), Lausanne, Cahiers Lausannois d'Histoire Médiévale, 41, 2007.

8. Torino, Archivio di Stato, corte, Ginevra, Categoria 1, paquet 7, n. 23 (46 feuillets). Le registre avait déjà été présenté et exploré dans L. Binz, «Les prédications "hérétiques" de Baptiste de Mantoue à Genève en 1430 », dans Pour une histoire qualitative. Études offertes à Sven StellingMichaud, Genève, 1975, p. 15-34. 


\section{La fresque contre le registre}

F. Morenzoni présente un document assez rare puisqu'il s'agit d'un dossier essentiellement constitué des interrogatoires menés en août 1430 par les notaires de Genève chargés par l'évêque - à la demande du procureur de l'accusé, Baptiste de Mantoue - de recueillir les témoignages des auditeurs du prédicateur afin d'invalider les vingt et un articles d'accusation formulés à son encontre par l'inquisiteur Ulric de Torrenté. Le livre comporte ainsi trois parties très inégales : la troisième et dernière partie - près des deux tiers de l'ouvrage est consacrée à l'édition de l'ensemble des pièces du registre, telles la requête du procureur ou les auditions des témoins, en version latine ainsi que dans une traduction en français (réalisée par Isabelle Jeger) voulue accessible au lecteur. La deuxième partie, très brève, présente le registre proprement dit et donne une reconstitution du calendrier de l'enquête.

C'est dans la première partie, qui sert d'introduction au dossier, que F. Morenzoni livre un commentaire sur l'affaire en la replaçant dans son contexte; il en présente les principaux protagonistes (le prédicateur, ses accusateurs, ses soutiens, les autorités concernées) et la chronologie, mettant en évidence les enjeux du procès; l'auteur en vient enfin à la défense de Baptiste de Mantoue en analysant les arguments avancés par le procureur dans la requête adressée à l'évêque, pièce maîtresse du registre. Dans ce commentaire introductif, en écho aux dépositions du dossier, on voit les prédications de Baptiste de Mantoue remporter un grand succès jusqu'à ce que le scandale éclate, du fait d'un autre prédicateur, le dominicain catalan Raphaël de Cardona, neveu supposé et disciple avéré de Vincent Ferrier. L'auteur tente de reconstituer les thèmes des sermons prononcés par Baptiste pendant son séjour, même si les témoignages des personnes auditionnées sont flous et lacunaires, et forcément orientés par le questionnaire.

F. Morenzoni s'attache à souligner le rôle pacificateur de l'évêque François de Metz qui cherche à mettre fin au conflit entre les deux prédicateurs et à ramener la paix dans sa cité, en évitant un procès, alors que la majorité des habitants, dont les franciscains, prend fait et cause pour Baptiste et que les dominicains genevois soutiennent Cardona. Le duc de Savoie intervient également dans le sens d'une conciliation, mais l'arrivée de l'inquisiteur Ulric de Torrenté, sans doute appelé en renfort par Raphaël de Cardona, va envenimer le conflit. Ce dernier entame alors une procédure judiciaire contre Baptiste de Mantoue. Malgré une seconde intervention du duc de Savoie en faveur d'une conciliation, l'inquisiteur s'entête; d'où l'ouverture de l'enquête, diligentée par l'évêque, sorte de contre-feu face à la procédure lancée par Ulric de Torrenté contre Baptiste de Mantoue. Ces «tribulations» permettent de faire la lumière sur les mécanismes spécifiques d'une affaire, mais c'est aussi l'occasion d'en 
savoir plus sur les hommes impliqués, à commencer par celui dont le nom figure dans le sous-titre du livre: Baptiste de Mantoue.

C'est un autre nom que J. Favier met en avant dans son ouvrage, mais, contrairement aux apparences, son livre n'est pas une biographie de Pierre Cauchon : celle-ci a déjà été proposée par François Neveux, l'historien spécialiste de la Normandie, qui suit l'évêque pas à pas, et développe la thèse selon laquelle Pierre Cauchon s'était efforcé de conduire avec une grande rigueur les interrogatoires de Jeanne d'Arc, en 1431, et d'appliquer les règles de procédure habituelles ${ }^{9}$. La vie de Cauchon n'en demeure pas moins le fil rouge de l'ouvrage de Favier. Et si le nom de Jeanne d'Arc figure aussi dans le titre, la procédure inquisitoriale menée contre la Pucelle n'occupe en fait qu'une petite partie du livre. En effet, l'objectif de l'auteur est de montrer comment la vie et les choix de celui qui fut le premier juge de Jeanne s'inscrivent dans un contexte complexe qu'il s'agit de reconstituer en une vaste fresque courant de la fin du XIV siècle à la fin du $\mathrm{Xv}^{\mathrm{e}}$ siècle.

La clé du livre est à chercher dans l'épilogue où l'auteur insiste sur le fait que la postérité et l'historiographie ont gardé l'image du «traître Cauchon», d'un évêque français à la solde des Anglais - comme s'il avait brusquement émergé du néant en tant qu'homme de paille n'attendant que le procès de Jeanne pour exister -, oubliant qu'il était universitaire. Or Cauchon et les autres membres du tribunal qui condamna la Pucelle avaient un passé. Tous sont porteurs d'une histoire que J. Favier se propose ici de raconter et d'éclairer, car elle a fabriqué ces juges. Ainsi, la première moitié du livre sert de long prélude au procès qui n'occupe en fait que la troisième partie - soit 116 pages sur un ouvrage qui en compte 725 ! - tandis que le second procès, en nullité, est envisagé au terme de ce long parcours dans le chapitre $\mathrm{XV}$, dernier acte de la cinquième partie intitulée «Des juges désavoués». J. Favier ne cherche pas tant à juger Cauchon ou à réparer une injustice (contrairement à $\mathrm{F}$. Neveux, qui a souvent cédé à cette tentation dans sa biographie de l'évêque de Beauvais) qu'à trouver un autre point de vue. Le sous-titre du livre suggère une démarche démonstrative censée résoudre le problème posé: comment Pierre Cauchon a-t-il pu devenir le juge de Jeanne d'Arc et, au-delà, comment les maîtres parisiens se sont-ils retrouvés impliqués dans ce célèbre procès? J. Favier choisit de faire de ce procès in materia fidei, non pas la source d'une histoire, mais l'aboutissement d'un long processus; il adopte le grand-angle, au risque d'écraser son sujet supposé et de le perdre de vue. L'entreprise est si ambitieuse qu'à vouloir embrasser tant de matière factuelle, l'auteur n'évite pas certains écueils et survole des points qu'il juge peut-être secondaires mais qui auraient mérité une réflexion plus approfondie

9. Voir F. Neveux, L'Évêque Pierre Cauchon, Paris, 1987. Cet ouvrage qui cherche à réhabiliter Pierre Cauchon fut couronné par l'attribution du prix Broquette-Gonin de l'Académie française. 
- telles des dispositions du droit canonique - alors qu'il s'attarde longuement sur certains épisodes, selon une logique événementielle.

En prenant de la hauteur, J. Favier a confondu une histoire personnelle avec l'histoire d'une Église en crise et d'un royaume déchiré par la guerre civile, une individualité avec une communauté, dans un style souvent dramatique qui soigne le décor, le prologue, les personnages de second plan et les multiples péripéties qui émaillent l'intrigue. D'ailleurs, s'il consacre plusieurs pages au début du livre à décrire la vie du Paris universitaire et à expliquer les rouages de l'enseignement scolastique, c'est pour mieux anticiper dans la procédure inquisitoire la fameuse réduction en articles, tant décriée pour son rôle joué au procès de Jeanne et mise en cause par l'avocat Maugier en 1455, suivant ainsi le fameux principe du «pistolet de Tchékhov ${ }^{10}$. Par ces procédés, J. Favier restitue cette fin du Moyen Âge qu'il connaît bien, en un livre foisonnant, où des idées souvent séduisantes permettent de tisser un canevas solide.

Beaucoup plus court, Le Prédicateur et l'Inquisiteur peut néanmoins refroidir les non-spécialistes. Même si la traduction en français facilite le maniement du registre en faisant d'ailleurs le choix d'alléger le style des notaires, le livre de F. Morenzoni est un travail d'universitaire qui s'adresse à d'autres historiens, tandis que J. Favier écrit à l'évidence pour un public, certes averti mais beaucoup plus large, de lecteurs férus d'histoire, d'amateurs de biographies et de passionnés de Jeanne d'Arc. En témoigne d'ailleurs l'absence de notes, jugées souvent rebutantes par les béotiens et donc superflues par les éditeurs, ce qui pose ici à l'historien le problème de nombreuses citations ou allusions non référencées tout au long de cette magistrale épopée. Sans être tout à fait un ouvrage de vulgarisation, ce Pierre Cauchon relève d'une école historique qui sort des normes académiques, et où l'imagination a sa part.

\section{Une justice de temps de crise}

En dépit de leurs différences, les deux livres s'articulent autour d'une inquisitio menée dans les années 1430. Dans les deux cas, il s'agit d'une justice extraordinaire impliquant des inquisiteurs, et dans les deux dossiers l'hérésie est invoquée. Par définition, l'inquisition relève de l'exception et traduit donc un bouleversement de l'ordre établi puisqu'il s'agit, par l'enquête et l'acte judiciaire (dont la production de registre écrit est une part intégrante et essentielle), de remettre les choses en ordre et de dire la norme; elle intervient dans ce qu'on pourrait appeler un «temps anormal» et révèle souvent les dérèglements, les fractures et les tensions d'une société à un moment donné. Par certains aspects, les

10. J. FAvIER, Pierre Cauchon ..., p. 609-10. 
deux affaires évoquées ici sont bien éloignées des dossiers instruits au XIII ${ }^{\mathrm{e}}$ siècle. Elles sont le reflet d'une autre époque. Il faut d'abord rappeler qu'au cours des $\mathrm{XIV}^{\mathrm{e}}$ et $\mathrm{XV}^{\mathrm{e}}$ siècles la procédure et la nature des tribunaux se sont compliquées puisqu'il est souvent arrivé aux papes d'instituer des commissions épiscopales pour mener des enquêtes in materia fidei, tandis que les officialités poursuivaient leur travail «ordinaire», y compris en cas d'hérésie quand l'inquisiteur faisait défaut. Ces commissions épiscopales investies du pouvoir délégué par le SaintSiège - souvent mises à contribution dans les procès d'évêques qui se sont multipliés au début du XIV ${ }^{\mathrm{e}}$ siècle et que la monarchie pontificale a utilisés comme mode de gouvernement de l'Église - représentent une autre facette de la justice extraordinaire, siégeant dans des tribunaux ad hoc, où les inquisiteurs sont en général absents ${ }^{11}$. Des inquisiteurs et des vice-inquisiteurs n'en sont pas moins nommés pendant toute la période, mais le concile de Vienne a imposé une cogestion des procès qui redonne à l'évêque un rôle de premier plan. L'Université de Paris se mêle également de la justice concernant hérésie et sorciers à partir de 1398 , ce qui ouvre la voie à ses interventions inquisitoriales du Xv $v^{e}$ siècle ${ }^{12}$. C'est dans ce contexte qu'il faut comprendre les deux affaires qui nous occupent, et les juges que mettent en scène les livres de F. Morenzoni et J. Favier.

11. C'est le cas de l'affaire Guichard de Troyes étudiée par F. Provost, Domus Diaboli... Voir aussi J. THÉRY : «Faide nobiliaire et justice inquisitoire de la papauté à Sienne au temps des Neuf: les recollectiones d'une enquête de Benoît XII contre l'évêque Donosdeo de'Malavolti (ASV, Collectoriae 61A et 404A)», dans S. Lepsius, T. Wetzstein éd., Als die Welt in die Akten kam. Prozeßschriftgut im europäischen Mittelalter, Francfort, 2008, p. 275-345 ; ID., Inquisitionis negocia. Les procédures criminelles de la papauté contre les prélats, d'Innocent III à Benoît XII (1198-1342). Première approche : aperçu sur les sources de la pratique, mémoire de fin de séjour à l'École française de Rome remis à l'Académie des Inscriptions et Belles-Lettres, 2004 [résumé du rapport fait sur ce travail par le Professeur André Gouron dans Académie des Inscriptions et Belles-Lettres. Comptes rendus des séances de l'année 2004, fasc. 4, Paris, 2006, p. 1617]; ID., Fama, enormia. L'Enquête sur les crimes de l'évêque d'Albi Bernard de Castanet (1307-1308). Gouvernement et contestation au temps de la théocratie pontificale et de l'hérésie des bons hommes, thèse de doctorat, Université Lumière-Lyon 2, 2003.

12. Le 19 septembre 1398, la Faculté de théologie de l'Université de Paris prononce une determinatio solennelle, dans laquelle sont condamnés vingt-huit articles relatifs à la magie et à la divination, puis, en 1413, sur ordre du roi, la Faculté de théologie doit se joindre à l'inquisition diocésaine pour une information concernant Jean Petit, de nombreux maîtres participant ainsi, aux côtés de l'évêque, de l'inquisiteur Jean Polet et du provincial des dominicains, à un «Concile de la Foi » chez l'official; en 1426, le pape encourage l'Université à conduire en matière de foi un procès engagé depuis deux ans par l'inquisition diocésaine contre Guillaume Vignier, puis, en 1428, Martin V reconnaît la compétence des maîtres dans les affaires d'hérésie qui surgiraient à Paris. Voir J.-P. Boudet, «Les condamnations de la magie à Paris en 1398 », Revue Mabillon, 12 (2001), p. 121157; ID., Entre science et nigromance. Astrologie, divination et magie dans l'Occident médiéval $\left(\mathrm{XIl^{e } - X V ^ { e }}\right.$ siècle), Paris, 2006. 
Ainsi, dans Pierre Cauchon, l'auteur s'efforce moins de faire le portrait de l'évêque de Beauvais que de décrire un certain milieu concerné par le désordre des temps et par l'inquisitio, celui de l'Université au tournant des XIV et $\mathrm{XV}^{\mathrm{e}}$ siècles. L'originalité de l'entreprise tient au fait d'envisager «le cas Cauchon» sous l'angle du monde des maîtres, et de la façon dont ils ont traversé les épreuves et les crises - ce que J. Favier appelle «la tourmente», qui va du déclenchement du Grand Schisme en 1378 à l'occupation anglaise et à la crise conciliaire. Il reconstitue alors, en une véritable geste des maîtres, une histoire chaotique qui a fait sortir les universitaires de leur rôle, en a fait des intellectuels engagés dans les affaires du temps, voire des juges. J. Favier rappelle que comme une grande majorité de ses pairs, maître Pierre a choisi le parti bourguignon, souvent mû par des aspirations réformatrices, et que ses choix l'ont mené au service des Anglais, jusqu'au conseil du roi Henri VI, et, de là, face à la Pucelle.

Il nous montre ainsi que le procès de Jeanne en 1431, véritable paroxysme de cette histoire tumultueuse, arrive précisément au moment où les maîtres sont en train de perdre le rôle de premier plan qu'ils avaient obtenu grâce au Grand Schisme, mais où ils conservent une place hors du commun et sont soucieux de défendre leurs prérogatives; un moment où Pierre Cauchon, qui fut naguère homme de confiance de l'Université et conseiller de Philippe le Bon, n'est plus que l'homme de Bedford, ce régent qui a fait sa fortune et pourrait tout aussi bien faire son malheur.

Dans la dernière partie qui traite des dernières années de la carrière de Pierre Cauchon et des suites de l'affaire, sur toile de fond du concile de Bâle et de victoire française, J. Favier rappelle enfin que de nouvelles logiques s'imposent à la fin du siècle, tandis que bon nombre des maîtres se sont ralliés à Charles VII, et que peu à peu l'Université est mise à l'écart puis réformée, retrouvant finalement sa fonction première de transmission des connaissances et de formation des esprits et des cadres de l'Église et de l'État. Ainsi, tous les grands moments $\mathrm{du} \mathrm{Xv}^{\mathrm{e}}$ siècle français sont évoqués, faisant tour à tour de Pierre Cauchon un témoin obligé (à l'ombre de plus illustres maîtres), un acteur de coulisses ou un protagoniste de premier plan - voire, après le 18 décembre 1442, un spectre silencieux - des différents drames qui se jouent, mais à force d'envisager par le menu chaque crise, controverse ou affrontement d'obédiences rivales, le propos initial en est quelque peu dilué au point que l'inquisitio concernant Jeanne ne semble parfois qu'un rebondissement parmi tant d'autres.

Le livre de F. Morenzoni consiste avant tout à publier un registre inédit. Le titre montre que la démarche se veut analytique et qu'il s'agit d'abord de passer une source judiciaire sous le microscope du chercheur. Néanmoins, dans la première partie, l'auteur prend soin de mettre le document en perspective. Il procède souvent par digressions, qui semblent d'abord éloigner de la procédure de 1430 mais tissent en fait, de chapitre en chapitre, tout un réseau de circonstances 
et de relations permettant d'éclairer l'affaire et de comprendre comment le prédicateur et l'inquisiteur se sont retrouvés face à face. Il rappelle en premier lieu les origines de ce moine italien, adepte de la stricte Observance de la règle bénédictine, et reconstitue son parcours entre 1411 et 1430 . C'est tout le petit monde des prédicateurs itinérants de l'époque qui est suggéré alors, tout comme les réactions que suscite en Italie le discours moralisateur et souvent eschatologique de ces hommes. En suivant les traces de Baptiste de Mantoue avant son arrivée à Genève, l'auteur rend compte des tensions qui traversent la Chrétienté et signale que Baptiste connut déjà quelques tribulations en 1418, le pape Martin V, pontife de la réconciliation après des décennies de Schisme, lui interdisant alors de prêcher. En 1421, Baptiste est même incarcéré à Venise. Après cette enquête, véritable «information préalable», qui permet de mieux cerner l'accusé et de donner un arrière-plan aux poursuites dont il fait l'objet, c'est au théâtre de l'affaire, la cité épiscopale de Genève, que l'auteur s'intéresse. Mais il n'en continue pas moins de relier la procédure à un contexte plus général. Ainsi, les sermons de Baptiste à Genève ont sans doute surtout traité de questions morales, mais le prédicateur a pu aussi aborder des sujets plus sensibles comme la Trinité et l'Eucharistie.

En effet, F. Morenzoni suggère - plus qu'il ne démontre - que l'affaire cristallise et révèle les divisions et les rivalités existant entre les prédicateurs, entre les ordres mendiants, mais aussi entre clergé régulier et séculier, voire au sein d'un même ordre religieux - l'augustin Andrea Biglia avait violemment critiqué Baptiste alors que le procureur du prédicateur en 1430 est aussi un frère augustin ; l'auteur souligne que le conflit entre Baptiste et les deux dominicains à l'origine de l'inquisitio s'exprime aussi à travers le mépris de deux «maîtres » en théologie envers un moine qui ne peut se prévaloir des mêmes grades universitaires mépris déjà affiché par le très cultivé Andrea Biglia qui traitait Baptiste de Mantoue de «moine ignorant». Toutefois, si F. Morenzoni esquisse une toile de fond utile, il le fait d'une façon plutôt impressionniste sans véritablement explorer les pistes qu'il découvre. Aussi, bien que les déboires de Baptiste de Mantoue ouvrent des perspectives et incitent au jeu d'échelles, l'arrière-plan ou les ramifications possibles de l'affaire restent allusifs, et il faut que l'analyse se resserre sur la prédication du moine, la personne de l'inquisiteur ou le contexte genevois pour que des logiques apparaissent plus nettement.

\section{Inquisitus et Inquisitor face à face}

Dans les deux ouvrages, le titre ou le sous-titre suggèrent que la relation entre le juge et l'accusé(e) et ce qu'elle met en jeu vont être abordés. On observe dans les deux cas qu'une parole est mise en cause, parce que publique et «populaire». Baptiste de Mantoue et Jeanne font sans aucun doute les frais 
de circonstances politiques qui dépassent leurs personnes, mais ils sont avant tout fauteurs de scandale au sens où l'entendait Thomas d'Aquin, c'est-à-dire un acte ou une parole qui peut provoquer la ruine spirituelle du prochain, qui peut être l'occasion d'une chute morale pour les autres. On remarquera que cette notion de scandale n'est pas éloignée de l'idée de contagion développée par Thomas d'Aquin à propos de l'hérésie qui, à elle seule, justifie la mise hors d'état de nuire - par le retour à l'orthodoxie ou par l'abandon au bras séculier - de ceux qui ont erré.

J. Favier, en évoquant le cas de deux femmes du peuple, dont Pierronne la Bretonne, condamnées à Paris pour hérésie avant le procès de Jeanne d'Arc, signale que la Pucelle «a fait école». Jeanne a pu se tromper, mais elle a surtout trompé ! Il rappelle aussi que Regnaut de Chartres, archevêque de Reims et partisan de Charles VII, a émis des réserves sur la Pucelle, et ce faisant est bien le porte-parole de ces prélats et docteurs qui n'aiment pas «qu'un chrétien - et de surcroît une laïque ignorante - se targue d'entretenir hors de la hiérarchie des relations directes et personnelles avec Dieu ${ }^{13}{ }^{»}$. Cauchon est un prélat qui peut partager ce point de vue, et qu'irritent l'insolence et la farouche détermination de celle qui comparaît devant lui. Ici, J. Favier aurait pu pousser plus loin la réflexion et dire que Cauchon et les autres maîtres ne pouvaient accepter de voir une simple fille s'emparer, par la grâce d'une supposée révélation, d'une matière réservée au saint des saints du monde universitaire, objet de raisonnements, de questions disputées et de longues années d'étude.

De fait, le juge du sous-titre, c'est bien sûr l'évêque de Beauvais mais aussi, selon la logique de l'ouvrage, tous ces docteurs qui sont amenés à se prononcer, en tant qu'assesseurs ou consultants, et donc à juger Jeanne. Au-delà des individus réputés pour leur savoir et leur sagesse, c'est enfin l'Université en tant que corps qui intervient en condamnant les douze articles censés résumer les fautes de la Pucelle. Des inquisiteurs «en titre», tous dominicains, sont néanmoins présents dans la procédure et mentionnés dans le livre de J. Favier; pourtant «le grand inquisiteur» de l'affaire Jeanne d'Arc reste l'évêque de Beauvais, et derrière ce juge ordinaire qui préside le procès, on trouve les maîtres parisiens qui réclament Jeanne dès sa capture, véritables juges extraordinaires sans être pour autant inquisiteurs. En revanche, Jean Le Maistre, vicaire de l'inquisiteur de France, et second juge du procès de Rouen, ne fait figure que de comparse. Malgré la perspective originale du livre, on regrettera que le passage sur l'inquisition et les inquisiteurs ne fournisse pas une pensée neuve et s'inscrive dans une tradition historiographique classique qui, d'une part, semble ignorer les décrets du concile de Vienne, et, d'autre part, continue de traiter l' «Inquisition » 
avec une majuscule, s'employant à invoquer une institution incontournable et influente qui «tend à élargir son domaine ${ }^{14}$ », mais sans jamais la montrer à l'œuvre! D'ailleurs J. Favier souligne bien que, lorsque le vice-inquisiteur Martin Billory écrit le 26 mai 1430 au duc de Bourgogne, c'est pour le compte de l'Université de Paris ${ }^{15}$, que Jean Graverent, inquisiteur de France, se dérobe, et que Jean Le Maistre, réticent dès le départ, finit par siéger au tribunal et constitue de fait «la caution théologique des universitaires, mais le juriste Cauchon fera peu de cas de son second», avant d'ajouter: «Lemaître est une doublure, voire un alibi $^{16}$.»

Tout comme Jeanne, le cas de Baptiste de Mantoue pose le problème d'une parole individuelle délivrée en public et à tous, par un clerc cette fois, mais hors du contrôle institutionnel et donc potentiellement subversive ${ }^{17}$. Il s'agit de faire taire ce prédicateur bénédictin, ce dont prétend d'abord se charger Raphaël de Cardona au début du sermon prononcé par Baptiste à Genève le 29 juin 1430. F. Morenzoni souligne au passage que le frère prêcheur devait être au courant des péripéties que le bénédictin avait connues dans le passé. Au cours des premières réunions chez l'évêque, Cardona formula un certain nombre d'accusations contre Baptiste que ce dernier réfuta en bloc, déclarant que tout ce qu'il avait prêché était conforme à la doctrine. Elles servirent probablement de base à la liste d'erreurs dressée par l'inquisiteur à son arrivée.

Le chapitre consacré à l'inquisiteur dominicain, Ulric de Torrenté, est particulièrement intéressant ${ }^{18}$. L'auteur revient sur le passé du juge et sur d'autres procédures concernant le dénommé Nicole Serrurier, autre prédicateur jugé hétérodoxe, qui avait été condamné pour hérésie à Tournai (mais avait abjuré ses erreurs), avant d'être arrêté quelques années plus tard à Lausanne où Ulric de Torrenté officiait comme inquisiteur. Dans le cadre de l'affaire de Lausanne, comme dans celle d'un autre procès à Fribourg, on voit un inquisiteur dont la marge de manœuvre est assez limitée, ce qui pose le problème des rapports de

14. Ibid., p. 379.

15. Ibid., p. 383.

16. Ibid., p. 425.

17. Les prédicateurs itinérants sont ainsi souvent poursuivis, dans la mesure où ils peuvent diffuser des idées fausses et participer à cette contagion qui caractérise le risque hérétique, d'autant qu'ils abordent des questions théologiques, sacramentelles ou ecclésiales susceptibles de faire débat. Or, si les controverses sur certains sujets sont éventuellement admises dans le cadre de disputes universitaires, il en va tout autrement de la prédication où la parole est publique. Voir à ce sujet M. Osterero, «Un prédicateur au cachot: Guillaume Adeline et le sabbat», Médiévales, 44 (2003), p. 73-96 (plus particulièrement p. 92).

18. Voir B. Andenmatten, K. Utz Tremp, «De l'hérésie à la sorcellerie: l'inquisiteur Ulric de Torrenté OP (vers 1420-1445) et l'affermissement de l'inquisition en Suisse romande», Revue d'histoire ecclésiastique suisse (Zeitschrift für schweizerische Kirchengeschichte), 86 (1992), p. $68-120$. 
force entre inquisiteur et ordinaire, et du rôle du pape dans cette dialectique. Dans l'affaire Baptiste de Mantoue, Ulric s'engage dans un véritable bras de fer avec l'évêque François de Metz, ce dernier étant bien décidé à isoler l'inquisiteur en le présentant d'abord comme simple coaccusateur. Le parcours d'Ulric de Torrenté ainsi que son attitude à Genève interrogent aussi ses relations avec le provincial de son ordre, dont la politique tient peut-être compte d'enjeux plus vastes. Franco Morenzoni souligne également que l'autorité d'Ulric de Torrenté sur le diocèse de Genève peut poser problème, la charge d'inquisiteur faisant l'objet d'un enjeu et donc d'un différend entre dominicains et franciscains, situation que le procureur de Baptiste de Mantoue ne manque pas d'exploiter. La question de juridiction et de compétence du juge est aussi soulevée dans le cas de Pierre Cauchon.

\section{Les enjeux politiques et religieux de l'inquisitio, la figure du juge indigne et le procès du procès}

En clamant que défendre la cause de Charles VII est une mission divine, Jeanne a laissé entendre que le choix du parti adverse (bourguignon d'abord, puis anglais) fut un choix contre Dieu, ce que ne peuvent admettre les clercs de l'Université puisque c'est précisément le parti qu'ils ont soutenu depuis des décennies. J. Favier montre que juger Jeanne est une nécessité, condamner ses paroles et surtout obtenir une rétractation représente une opportunité pour les maîtres, dont Cauchon qui, pour être devenu homme d'État au service de Lancastre, n'en est pas moins homme de foi et prélat. Or les Anglais veulent avant tout la mise à mort de la sorcière, et Cauchon est aux ordres.

Le caractère politique des procès de Jeanne d'Arc est une évidence, de nombreux ouvrages l'ont montré, le redire n'a rien de novateur. J. Favier rappelle que l'organisation de la procédure se décida au conseil du roi Henri VI, dont Pierre Cauchon faisait partie, et que la condamnation à mort fut imposée par les Anglais, furieux de voir «la sorcière» s'en sortir au prix d'une abjuration dont les juges pouvaient au contraire s'estimer satisfaits. C'est là que J. Favier innove davantage, soulignant que les intérêts des juges et des Anglais divergent à la fin de la procédure, que leurs perspectives se heurtent. Il insiste même sur le «drame personnel ${ }^{19} »$ des maîtres qui cherchent avant tout à se justifier d'un passé complexe, des juges pour qui il n'est pas nécessaire que Jeanne meure pour qu'ils aient été du côté du droit, de l'Église et de Dieu. Il suffit seulement qu'elle se désavoue, point de vue que ne peuvent partager le comte de Warwick et le duc de Bedford qui, eux, sont en guerre et veulent la victoire. Aux yeux des maîtres, 
il faut surtout que Jeanne «réduise à néant les dires des fidèles de Charles VII», ce qui d'ailleurs écarte toute possibilité de rançon puisque le paiement d'une rançon ne discrédite en rien le parti du rançonné ${ }^{20}$; il s'agit pour ceux qui ont adhéré si longtemps aux choix politiques de Jean sans Terre puis de Philippe le Bon de rendre légitime le déni opposé aux Armagnacs, puis à Charles VII. Le 24 mai au cimetière de Saint-Ouen, Jeanne craque enfin et se rétracte, admettant qu'elle a été schismatique. Selon J. Favier, Cauchon et les docteurs ont gagné alors que pour les Anglais la défaite est complète. Mais Cauchon, qui joue sa carrière et a subi les reproches de Bedford et Warwick, adopte alors le point de vue des Anglais. On connaît la suite. Sans être dupe des témoignages enregistrés au procès de 1456,1 'auteur considère que la reprise des habits d'homme ne peut avoir été le fait du hasard, que Jeanne ait eu ou non le choix, car le relaps était le plus sûr moyen de donner satisfaction au régent, et cela ne put se faire à l'insu de l'évêque de Beauvais. Il est intéressant de voir cependant que J. Favier dépeint un Cauchon angoissé, servant le Lancastre et menant à terme la procédure qui condamne Jeanne comme relapse, mais ne maîtrisant plus vraiment les événements. L'auteur explique en outre la fameuse «Information Posthume», que les notaires refusèrent d'authentifier, par l'inquiétude qui hanterait encore l'évêque de Beauvais après le bûcher. J. Favier semble alors connaître les tréfonds de l'âme, ou du moins l'intimité psychique, de Pierre Cauchon quand il affirme : «Depuis le début il ne pense qu'à une chose, et ce n'est pas à la cascade des échecs anglais. Ce sont les voix. Derrière l'acharnement de Cauchon perce toujours l'inquiétude personnelle. Qui est contre Dieu ? [...] Et, surtout, il voudrait que Jeanne ait avoué avoir inventé ses voix ${ }^{21}$.» Le style de l'ouvrage, il est vrai, se prête à l'exercice médiumnique.

Jusqu'au bout, J. Favier a voulu lier le sort de l'évêque de Beauvais à celui des maîtres et, s'il ne renie pas l'homme d'État qu'est devenu Pierre Cauchon, ni même le fait qu'il s'est détaché finalement de l'Université - voire a trahi ses anciens collègues ${ }^{22}$ - en entrant au service de Bedford, il le montre comme un clerc soucieux de l'institution ecclésiale, ambitieux mais non pas cynique, un chrétien qui certes n'a jamais fini ses études de théologie mais pour qui la question du salut reste essentielle. En somme, Pierre Cauchon aurait incarné les engagements, les interrogations, les aspirations et les dérives d'une époque.

20. Ibid., p. 455 .

21. Ibid., p. 475.

22. Pierre Cauchon aurait joué sur deux tableaux, prétendant être le représentant de l'Université de Paris auprès du duc de Bourgogne quand, en fait, il réclamait la prisonnière pour lui-même car Jeanne avait été prise dans son diocèse, et il préparait sans doute le rachat de la captive par les Anglais. Ibid., p. 383-391. 
Dans le cas de Baptiste de Mantoue, les aspects politiques, pour être moins évidents, n'en sont pas moins présents. Dans son commentaire, F. Morenzoni se propose de comprendre pourquoi l'évêque et le duc ont tous deux œuvré pour apaiser le conflit. Des enjeux politiques peuvent avoir influencé l'attitude prudente des deux hommes. L'auteur révèle alors les coulisses du dossier, où les tribulations du prédicateur apparaissent comme une affaire assez mineure, en marge d'une partie serrée où se joue l'exercice de la souveraineté à Genève et où, bien qu'ayant tous deux intérêt à calmer le jeu dans l'affaire Baptiste, le duc et l'évêque sont des adversaires et non des alliés. En effet, Amédée VIII, qui a conçu le projet d'intégrer la cité à ses possessions, manœuvre depuis plus de dix années dans ce sens au moment où Baptiste de Mantoue prêche à Genève ${ }^{23}$. Alors que le pape semble favorable à un plan de partage de la ville, le duc peut se croire sur le point de concrétiser son projet. Il doit cependant éviter tout faux pas et ne pas se mettre à dos les bourgeois de Genève qui soutiennent Baptiste. L'évêque doit de son côté prouver qu'il est capable de rétablir l'ordre et la paix publique d'autant que l'un des arguments du duc est que François de Metz est défaillant tant pour la gestion de la cité que pour sa sécurité. L'évêque doit en outre éviter toute intervention extérieure, et plus précisément celle d'un inquisiteur susceptible de demander l'aide du bras séculier, c'est-à-dire le pouvoir ducal! En autorisant une contre-enquête, il défend ses prérogatives et peut encore espérer tuer dans l'œuf la procédure inquisitoriale lancée sans son aval par l'inquisiteur, ou du moins éviter de passer pour un ennemi de Baptiste aux yeux des bourgeois de la ville. L'évêque reste cependant prudent, comme le prouve l'existence d'un acte biffé mais conservé dans le registre, par lequel il s'engageait à prononcer un jugement, et auquel fut substitué un nouvel acte épiscopal qui laissait dans le flou le but de l'enquête.

Avant de conclure sur l'épilogue de l'affaire Baptiste de Mantoue, F. Morenzoni analyse la requête que Jean Chauvin, juriste et procureur du prédicateur, envoya à l'évêque, et qui constitue la première pièce du registre. Chauvin s'emploie à démontrer que les accusations proférées par les dominicains sont fausses et que la bona fama de Baptiste de Mantoue ne peut faire de doute ; il met surtout en évidence le caractère arbitraire et partial du procès lancé par l'inquisiteur et insiste sur toutes les irrégularités commises. Toute la procédure étant viciée, elle doit être frappée de nullité. Les témoins n'hésitent pas, de leur côté, à critiquer l'acharnement, voire la mauvaise foi, des deux dominicains . Baptiste de Mantoue aurait pourtant été condamné, en 1431 ou 1432, les dominicains en ayant appelé au pape Martin V. Néanmoins en 1440, Ludovico

23. Dans Le Prédicateur et l'Inquisiteur... (n. 181, p. 64), Franco Morenzoni signale que le dossier concernant les démarches de l'évêque est toujours conservé à Turin et qu'il compte consacrer une étude approfondie à cet ensemble documentaire. 
Barbo, qui fut le supérieur de Baptiste à Santa Giustina et le légat du pape Eugène IV au concile de Bâle, rédige un texte comprenant un éloge de Baptiste dont la renommée ne semble pas avoir été entachée au sein de la congrégation bénédictine de la stricte Observance, ce qui pose la question de la portée d'une telle condamnation si elle a bien eu lieu.

Dans le cas de Jeanne d'Arc, un nouveau procès est ouvert en 1455, un peu plus de dix ans après la mort de Pierre Cauchon, cette fois pour casser la condamnation de 1431. Il est frappant de voir que, comme dans le cas de Baptiste, les juges de $1456 \mathrm{~s}$ 'attaquent à une autre procédure et s'efforcent d'en démontrer la nullité. Des termes et des arguments similaires s'y retrouvent comme si le registre de 1430 avait servi de modèle occulte et de jurisprudence indicible, étant bien entendu que Baptiste n'est pas encore condamné au moment où ce registre est établi alors que la condamnation de Jeanne a, elle, été consommée depuis longtemps quand la procédure en nullité est enclenchée au début des années 1450. Dans les deux cas un renversement s'opère faisant du juge un accusé, d'autant que pour la condamnation de la Pucelle, les juges ainsi mis en cause par les plaignants sont déjà morts, ce qui donne à la procédure de 1455-1456 des allures de procès posthume et de procès en contumace. Jean Bréhal, docteur en théologie et inquisiteur de France, mène alors la charge contre l'évêque de Beauvais qui fera désormais figure de clerc indigne et de serviteur de l'étranger ${ }^{24}$. J. Favier s'accorde avec ses prédécesseurs pour dire que cette fois les rôles sont inversés et les juges épiscopaux apparaissent comme les assesseurs de l'inquisiteur. L'auteur, qui suit F. Neveux, souligne que Bréhal, loin d'être impartial, s'emploie à alléger les charges qui pèsent sur les dominicains de 1431, en particulier sur le vice-inquisiteur, Jean Le Maistre. On remarque de nouveau la solidarité au sein de l'ordre des Prêcheurs. Ceux qui se sont ralliés au roi de France ne sont pas inquiétés et l'Université est exonérée. Le seul juge qui demeure en pleine lumière et contre lequel Jean Bréhal prononce un véritable réquisitoire, c'est Pierre Cauchon. Comme beaucoup d'auteurs l'ont dit, il s'agit moins, en 1456, d'innocenter Jeanne d'Arc que de légitimer Charles VII. Pour J. Favier, la révision du procès de condamnation est aussi l'occasion d'offrir à l’Église une catharsis qui purge de leurs fautes tant de clercs, prélats et docteurs.

24. Après avoir étudié il y a quelques années la question des inquisiteurs de la France du Nord du XIII ${ }^{\mathrm{e}}$ au XV $\mathrm{x}^{\mathrm{e}}$ siècle (mémoire de D.E.A., Paris I-Panthéon Sorbonne, dir. C. GAuvarD) selon une approche plutôt prosopographique, j'ai entrepris une thèse de doctorat (en cours), sous la direction de Dominique Iogna-Prat, sur Jean Bréhal, inquisiteur apparemment atypique, personnage peu connu et pourtant déterminant dans la procédure en nullité. 


\section{Pour la refonte d'une histoire de l'inquisitio médiévale}

L'histoire de l'inquisition médiévale a d'abord été écrite, aux XIX et $\mathrm{XX}^{\mathrm{e}}$ siècles, par des érudits et des chercheurs, en particulier grâce aux travaux portant sur la collection Doat, c'est-à-dire à partir de sources concernant les enquêtes et procès menés dans le Sud du royaume de France, à Pamiers, Toulouse ou encore Carcassonne, bases sur lesquelles on a ensuite généralisé 25 . Inversement, une histoire du Saint-Office a été racontée depuis la perspective de l'Inquisition romaine fondée au moment de la Contre-Réforme ${ }^{26}$, en suivant une trame linéaire et donc en intégrant les procès et les juges du Moyen Âge dans un ensemble relativement cohérent, avec toutefois des phases d'intensité, voire d'apogée, ou à l'inverse de supposé déclin de l'institution - d'autant qu'à la fin du XVI ${ }^{\mathrm{e}}$ siècle, c'est un manuel composé par un inquisiteur catalan au XIV siècle que Rome choisit comme référence commune à tous les inquisiteurs pontificaux de la Chrétienté ${ }^{27}$. L'inquisition médiévale devait dès lors logiquement en constituer un prototype ou un embryon. Cette projection sur les siècles antérieurs d'une institution qu'on a voulue centralisée et organisée de façon rigoureuse et uniforme, tout comme la tendance à ériger les tribunaux méridionaux - voire espagnols - en archétypes de fonctionnement de l'inquisition médiévale, ont longtemps brouillé les pistes. Quand on y regarde de plus près, on constate pourtant que le cadre géographique et la période changent souvent la donne, et que l'inquisitio médiévale des $\mathrm{XIV}^{\mathrm{e}}$ et $\mathrm{XV}^{\mathrm{e}}$ siècles est une réalité multiforme et mobile, qui s'écarte souvent des modèles de référence et échappe aux carcans rigides. Il faut désormais envisager l'inquisition dans cette perspective et remettre en cause l'usage de la majuscule dont tant d'historiens peinent à se défaire. Dans un article décisif, paru en 1995, Richard Kieckhefer a pourtant ouvert la voie à une recherche décomplexée, en avançant que l'expression officium inquisitionis, au Moyen Âge, renvoyait à une fonction plus qu'à un office institutionnel ${ }^{28}$. Ainsi, au niveau régional, la souplesse et l'adaptation sont souvent de mise, et

25. Voir en particulier la synthèse incontournable de H. C. LEA, Histoire de l'Inquisition au Moyen Âge, Paris, 1900-1902, rééd. Grenoble, 1986. Henry Charles Lea fut aussi l'auteur d'une History of the Inquisition of Spain, New York, 1906.

26. C'est-à-dire la Sacrée congrégation de l'inquisition romaine et universelle instituée par le pape Paul III dans la bulle Licet ab initio, le 21 juillet 1542.

27. N. EyMERIC, Directorium inquisitorum, Rome, 1587 (réédition avec glose).

28. R. KIECKHEFER, «The Office of Inquisition and Medieval Heresy: the Transition from Personal to Institutional Jurisdiction », Journal of Ecclesiastical History, 46 (1995), p. 36-61. Au cours de mes propres recherches sur les inquisiteurs en France du Nord aux XIII ${ }^{\mathrm{e}} \mathrm{XIV}^{\mathrm{e}}$ et XV siècles, j'ai pu constater à quel point ses hypothèses pouvaient se confirmer. L'étude des mandats et des activités de ces inquisiteurs, la diversité des situations observées, la flexibilité des affectations, le nombre de vacances successives et récurrentes pour certains diocèses tendent à remettre en cause l'idée d'une organisation structurée de façon globale ou d'une institution établie. 
l'inquisition paraît, non pas organisée ou systémique, mais plutôt protéiforme ${ }^{29}$. L'inquisiteur, loin d'être une figure toute-puissante suscitant l'effroi, doit tenir compte d'autres acteurs dans un contexte à la fois local et global, quand il n'est pas même tout simplement relégué au rôle de «second couteau», et seuls des hommes déterminés, bénéficiant de réseaux puissants, voire de protecteurs, peuvent espérer imposer leurs vues. Les deux ouvrages évoqués ici concourent, dans des styles très différents et sans épuiser le sujet, à le dire, et nous invitent à explorer plus avant les rapports de force entre les juges à l'occasion des procès dont les sources nous ont gardé la trace ou l'écho, à mieux comprendre la part que jouent les réseaux et les influences «extérieures», tant laïques que cléricales ou pontificales, et enfin à mesurer le rôle de la politique interne des ordres mendiants, en particulier chez les dominicains, véritables professionnels de l'inquisitio. Plus que jamais ces regards croisés révèlent le danger de certains paradigmes et insistent sur la nécessité d'appréhender ces questions à différentes échelles.

Laurence SiLVESTRE - LAMOP, 17, rue de la Sorbonne, 75005 Paris

29. Voir à ce sujet, dans Praedicatores, Inquisitores..., deux contributions éclairantes, fondées sur une étude régionale: P. KRAS, «Dominican Inquisitors in Medieval Poland», p. 249-287 et K-B. SPRINGER, «Dominican Inquisition in the Archdiocese of Mainz (1348-1520)», p. 311-393; voir aussi M. M. TAvuZZI, Renaissance Inquisitors : Dominican Inquisitors and Inquisitorial Districts in Northern Italy, 1474-1527, Leiden, 2007. 
